\title{
Role of Hydration in Contrast-Induced Nephropathy in Patients Who Underwent Primary Percutaneous Coronary Intervention A Meta-Analysis of Randomized Trials
}

\author{
Zhuoqun Wang, ${ }^{1} \mathrm{MD}$, Yunping Song, ${ }^{1} \mathrm{MD}$, Geru A, ${ }^{1} \mathrm{MD}$ and Yongle $\mathrm{Li},{ }^{1} \mathrm{MD}$
}

\begin{abstract}
Summary
Patients with ST-segment elevation myocardial infarction (STEMI) who are treated by primary percutaneous coronary intervention (PPCI) have an increased risk of developing contrast-induced nephropathy (CIN) when compared with patients undergoing elective percutaneous coronary intervention (PCI). However, CIN prevention measures are less frequently applied in PPCI than in elective PCI. At present, no preventive strategy has been recommended by the current guidelines for patients with STEMI undergoing PPCI.

Published research was scanned by formal searches of electronic databases (PubMed, EMBASE, and the Cochrane Central Register of Controlled Trials) from 1966 to July 2018. Internet-based sources of information on the results of clinical trials in cardiology were also searched.

A total of three randomized trials involving 924 patients were included in the present meta-analysis, of whom 462 received hydration with isotonic saline (hydration group) and 462 received no hydration (control group). Periprocedural hydration with isotonic saline was associated with a significant decrease in the rate of CIN (16.9\% in the hydration group versus $26.4 \%$ in the control group; summary risk ratio: $0.64,95 \%$ confidence interval: $0.50-0.82, P=0.0005)$. There was no difference in the rate of postprocedural hemodialysis or death between the groups.

Intravenous saline hydration during PPCI reduced the risk of CIN without significantly altering the rate of requirement for renal replacement therapy or mortality.
\end{abstract}

Key words: Myocardial infarction, Intravenous saline hydration

(Int Heart J 2019; 60: 1077-1082)

$\mathrm{P}$ atients with ST-segment elevation myocardial infarction (STEMI) who are treated by primary percutaneous coronary intervention (PPCI) have an increased risk of developing contrast-induced nephropathy (CIN) when compared with patients undergoing elective percutaneous coronary intervention (PCI). ${ }^{1)}$ However, CIN prevention measures are less frequently applied in PPCI than in elective PCI. ${ }^{2)}$ Although several specific pharmacological interventions have been proposed for the protection of the renal function, compared with intravenous volume expansion with isotonic saline, hydration with $\mathrm{N}$ acetylcysteine (NAC), sodium bicarbonate $\left(\mathrm{NaHCO}_{3}\right)$, or combined $\mathrm{NAC}$ and $\mathrm{NaHCO}_{3}$ does not significantly reduce the incidence of CIN. ${ }^{3,4}$ At present, no preventive strategy has been recommended in the current guidelines for patients with STEMI undergoing PPCI. ${ }^{2.5)}$ The purpose of this meta-analysis was to evaluate the possible beneficial role of periprocedural intravenous volume expansion with isotonic saline in patients with STEMI undergoing PPCI.

\begin{abstract}
Methods
All analyses were based on previously published studies; thus, no ethical approval or patient consent was required.

Literature search and study selection: In this study, we searched randomized trails concerning CIN prevention in PPCI through electronic databases (PubMed, EMBASE, and the Cochrane Central Register of Controlled Trials) from 1966 to July 2018 using the following medical subject heading terms: nephropathy, isotonic saline, and hydration. We also scanned Internet-based sources of information on the results of clinical trials in cardiology (htt p://www.theheart.org, http://www.cardiosource.com/clinical trials, http://www.clinicaltrialresults.com, and http://www.t ctmd.com). Other sources included conference proceedings from the American College of Cardiology, American Heart Association, and European Society of Cardiology meetings. Besides, we also searched relevant reviews and editorials, which were published in major medical journals within the last year, assessing for possible information of
\end{abstract}

From the ${ }^{1}$ Department of Cardiology, Tianjin Medical University General Hospital, Tianjin, China.

This research was supported by grants from the Technology Research and Development Program of Tianjin (15JCYBJC28000).

Address for correspondence: Yongle Li, MD, Department of Cardiology, Tianjin Medical University General Hospital, Tianjin Medical University, Tianjin, 300052, China. E-mail: liyongle@aliyun.com

Received for publication December 27, 2018. Revised and accepted March 29, 2019.

Released in advance online on J-STAGE August 23, 2019.

doi: 10.1536/ihj.18-725

All rights reserved by the International Heart Journal Association. 


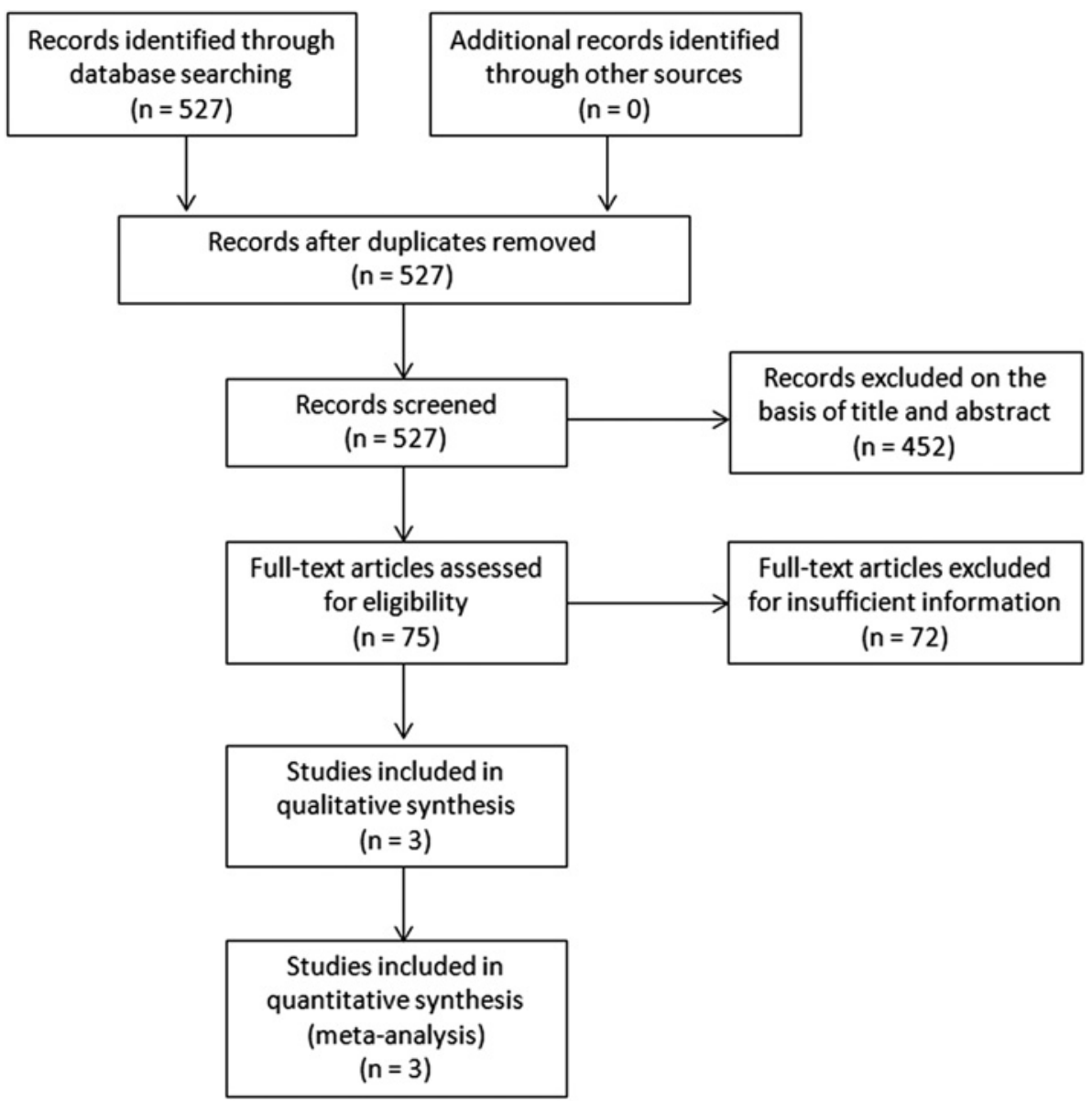

Figure 1. Flowchart of the study selection procedure.

interest. Regardless of the language or publication form, all relevant studies were considered eligible for this study. The most complete and/or recent data were used if there were a variety of reports from the same trial. ${ }^{6}$

Study selection and outcome measures: Eligible studies had the following characteristics: (1) they were randomized trials of any duration for the prevention of CIN, (2) the intervention was hydration with isotonic saline, (3) subjects (with or without preexisting kidney disease) underwent PPCI, and (4) the studies reported the outcome events (including no events) in both arms.

The primary outcome measure was the incidence of CIN, defined as an increase of serum creatinine level greater than $25 \%$ or greater than $0.5 \mathrm{mg} / \mathrm{dL}$ from baseline within 72 hours after contrast administration. ${ }^{7)}$ The secondary outcome measures included (1) the need for renal replacement therapy and (2) in-hospital mortality.

Data extraction: Using a standardized protocol and reporting form, three investigators (ZQ, YP, and GR) independently extracted data on the patients and study characteristics, outcomes, and study quality for each trial. ${ }^{6}$ A fourth reviewer (YL) resolved disagreements by consensus.

Statistical analysis: Data were analyzed through an intention-to-treat protocol using Review Manager
(RevMan) 5.0.16.0. In order to estimate the pooled relative risk (RR), ${ }^{6}$ we used the Mantel-Haenszel method for fixed effects and the DerSimonian-Laird method for random effects. Q-statistics were used to test the heterogeneity of the included studies, and the $\mathrm{I}^{2}$ statistics were used to test the extent of inconsistency between the results. When heterogeneity between studies was absent, the Mantel-Haenszel and DerSimonian-Laird methods produced very similar results. Since there were a small number of events, we reported fixed-effects estimates for more robust meta-analysis calculations. Funnel plot analysis was used to assess the possibility of publication bias. Data were presented as RRs with 95\% confidence intervals (CIs). $P<0.05$ was considered statistically significant.

\section{Results}

Search results and study characteristics: The initial search yielded 527 results, of which three studies (two prospective randomized clinical trials ${ }^{2,8)}$ and one subgroup of one randomized clinical trial ${ }^{9)}$ ) met our inclusion criteria. Figure 1 shows the flowchart of this procedure. The studies included a total of 924 patients undergoing primary PCI for STEMI, of whom 462 received hydration with isotonic saline (hydration group) and 462 received no 
hydration (control group). The baseline characteristics of the patients enrolled in the individual trials are listed in the Table.

CIN: The overall incidence of CIN was $21.6 \%$ (200 out of 924): $16.9 \%$ (78 out of 462) in the hydration group and $26.4 \%$ (122 out of 462) in the control group. Preprocedural hydration with isotonic saline significantly decreased the risk of CIN compared with no hydration (RR: 0.64; 95\% CI: 0.50-0.82; $P=0.0005$ ) (Figure 2). There was no significant heterogeneity between trials $(P=0.26)$.

Need for renal replacement therapy: There was no difference in the need for renal replacement therapy between the hydration group and the control group in the three trials, with 3 out of 462 patients in the hydration group $(0.6 \%)$ and 10 out of 462 patients in the control group $(2.2 \%)$ requiring renal replacement therapy. The pooled RR was 0.30 (95\% CI: 0.08-1.08, $P=0.07$ ) (Figure 3). There was no significant heterogeneity between trials $(P=$ $0.50)$.

In-hospital mortality: There was no difference in the mortality rate between the hydration group and the control group in the three trials, with 15 out of 462 deaths in the hydration group $(3.2 \%)$ and 26 out of 462 deaths in the control group (5.6\%). The pooled RR was 0.58 (95\% CI: $0.31-1.08, P=0.08$ ) (Figure 4$)$. There was no significant heterogeneity between trials $(P=0.42)$.

\section{Discussion}

We conducted a meta-analysis on the role of isotonic hydration in preventing CIN in patients who underwent PPCI. We found three randomized controlled trials that met our criteria. In this meta-analysis, we found that preprocedural hydration with isotonic saline significantly reduced the incidence of CIN compared with no hydration, without decreasing the incidence of death or need for hemodialysis.

Patients with STEMI treated by PPCI have an increased risk of developing CIN when compared with patients undergoing elective PCI, which is associated with higher rates of early clinical complications and mortality. ${ }^{9)}$ In patients undergoing elective PCI, worse cardiac and renal function may be associated with an increased risk of developing CIN. ${ }^{10)}$ Similarly, conditions such as hemodynamic instability and impaired systemic perfusion caused by left ventricular dysfunction, large volume of contrast medium, renal hypotension, and lack of hydration or other effective prophylactic measures may contribute to the development of renal dysfunction in patients with STEMI undergoing PPCI. ${ }^{1)}$ At present, since no feasible, effective therapeutic strategy for CIN has been identified, certain preventive measures against CIN can be adopted in a timely manner in patients with STEMI undergoing PPCI to improve renal and cardiac outcomes. ${ }^{9)}$

Routine administration of hydration protocols before contrast exposure is an established preventive measure for preventing CIN in patients with chronic renal failure undergoing elective PCI. ${ }^{2,9}$ However, no optimal hydration strategy has been well established in patients undergoing PPCI. Earlier, smaller reports have probably overestimated the magnitude of the benefit of $\mathrm{NaHCO}_{3},{ }^{2,11,12)}$ whereas larger, more recent studies have produced neutral results. ${ }^{2,3,13,14)}$ In the recent Prevention of Contrast-Induced Nephropathy with N-Acetylcysteine or Sodium Bicarbonate in Patients With ST-Segment Myocardial Infarction (CINSTEMI) trial, Thayssen, et al. ${ }^{3)}$ randomized 720 patients in a $1: 1: 1: 1$ ratio to receive hydration with sodium chloride together with one out of four prophylactic regimes: (1) NAC, (2) $\mathrm{NaHCO}_{3}$ infusion, (3) NAC in combination with $\mathrm{NaHCO}_{3}$, or (4) hydration with sodium chloride infusion alone. CIN occurred in 141 patients $(21.9 \%)$. Preventive treatment with $\mathrm{NAC}, \mathrm{NaHCO}_{3}$, or NAC and $\mathrm{NaHCO}_{3}$ combined did not significantly reduce the rate of CIN compared with hydration via intravenous infusion of sodium chloride alone. In our study, intravenous saline hydration during PPCI achieved a $36 \%$ reduction in the risk of CIN relative to nonhydration. Given the higher incidence of CIN, morbidity, and mortality in patients undergoing PPCI, preventive intravenous saline hydration should be mandatory for these patients unless otherwise contraindicated. ${ }^{2)}$ Recently, Lancet published the Prophylactic hydration to protect renal function from intravascular iodinated contrast material in patients at high risk of contrast-induced nephropathy (AMACING trial), which showed that nonprophylaxis is noninferior when compared with intravenous hydration. ${ }^{15)}$ However, in the AMACING trial, emergency and intensive care patients were excluded from the study population. The result, therefore, could not be generalized to include such cases. Aggressive hydraTion in patients with ST-Elevation Myocardial infarction undergoing Primary percutaneous coronary intervention to prevenT contrast-induced nephropathy (ATTEMPT) is an ongoing multicenter, randomized, controlled clinical trial assessing the effect of aggressive hydration with normal saline guided by the left ventricular end-diastolic pressure compared with general hydration on CIN and important clinical outcomes in patients after STEMI. ATTEMPT will provide reliable evidence to guide the optimal hydration strategy for patients with STEMI undergoing primary PCI. ${ }^{4)}$

In our study, we did not find any differences in the need for renal replacement therapy or in-hospital mortality between the groups, which could be attributed to the lack of power of the included studies. The studies that were included in our meta-analysis were designed to focus on the incidence of CIN rather than patient-centered outcomes. According to the current data and our study results, we speculated that this reduction in CIN may translate into long-term benefits. However, more studies are necessary for further evaluation.

Our study has several potential limitations. First, there was a significant crossover rate between the two treatment groups. This issue was an expected limitation because the protocol allowed the physician to change patients between groups according to the clinical or hemodynamic situation. We performed an intention-to-treat analysis to reduce this limitation. Second, all trials were single-center studies without blinding. Third, the number of eligible trials was relatively small, which might have limited the power of detecting publication bias. Finally, the total number of patients was not large, limiting the ability to detect meaningful differences in secondary out- 


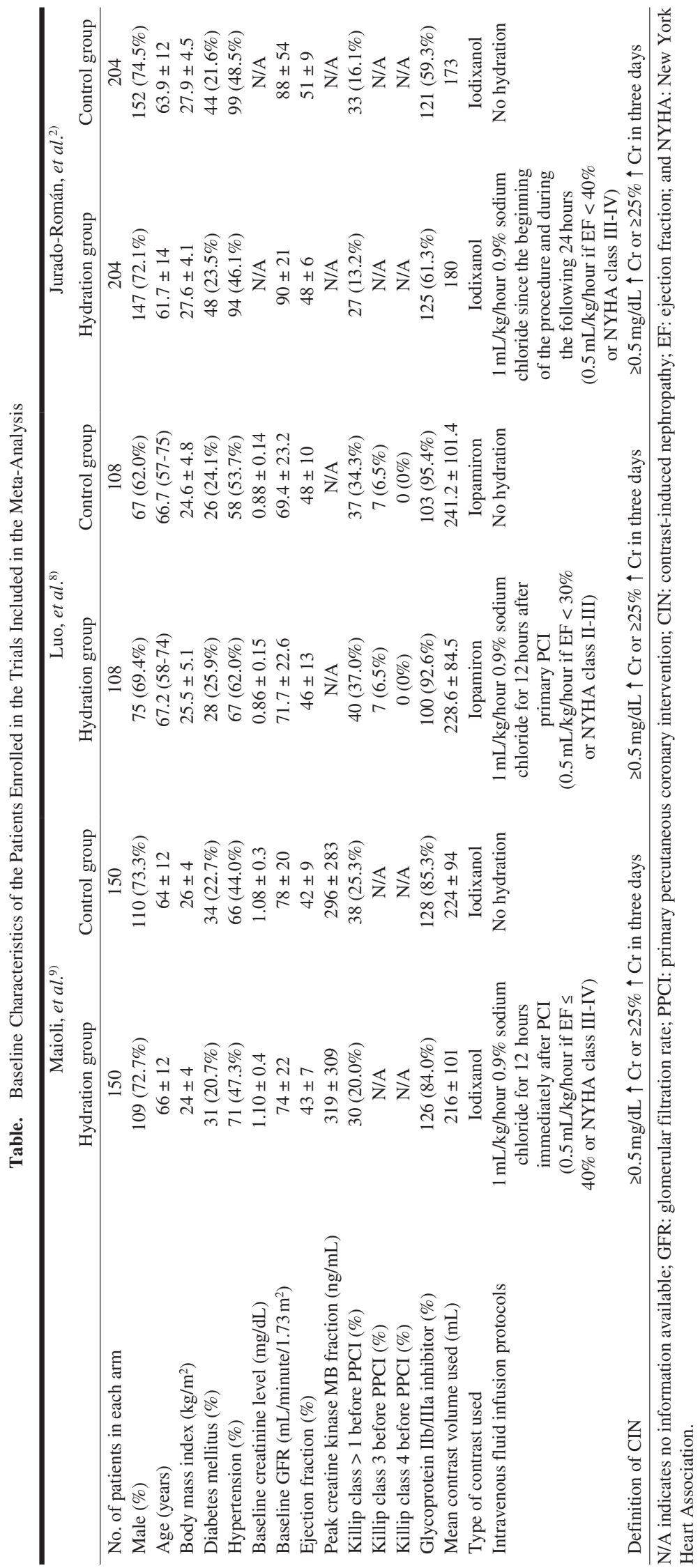




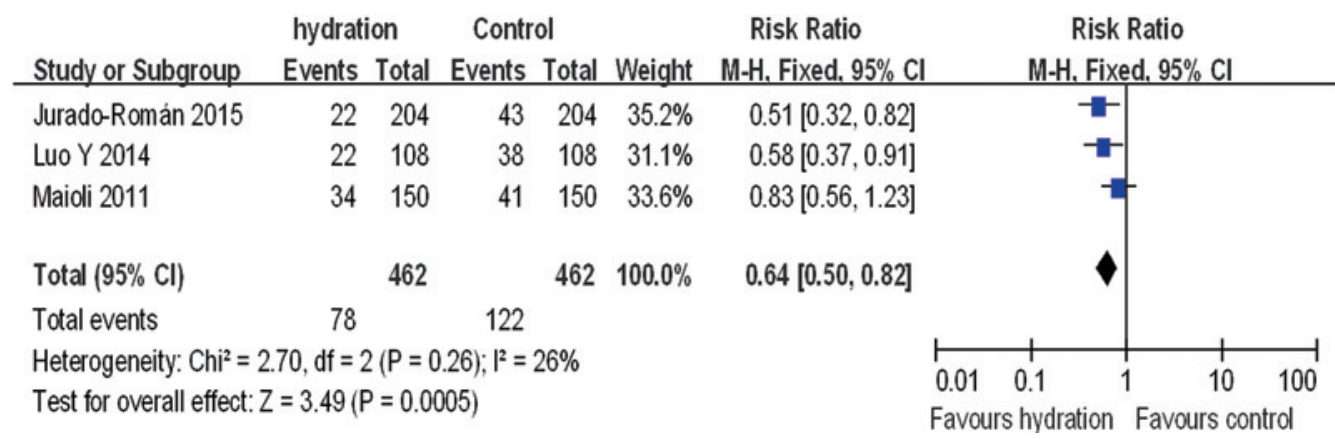

Figure 2. Relative risk (RR) for contrast-induced nephropathy (CIN) in patients who received either hydration with isotonic saline or no hydration. CI indicates confidence interval.

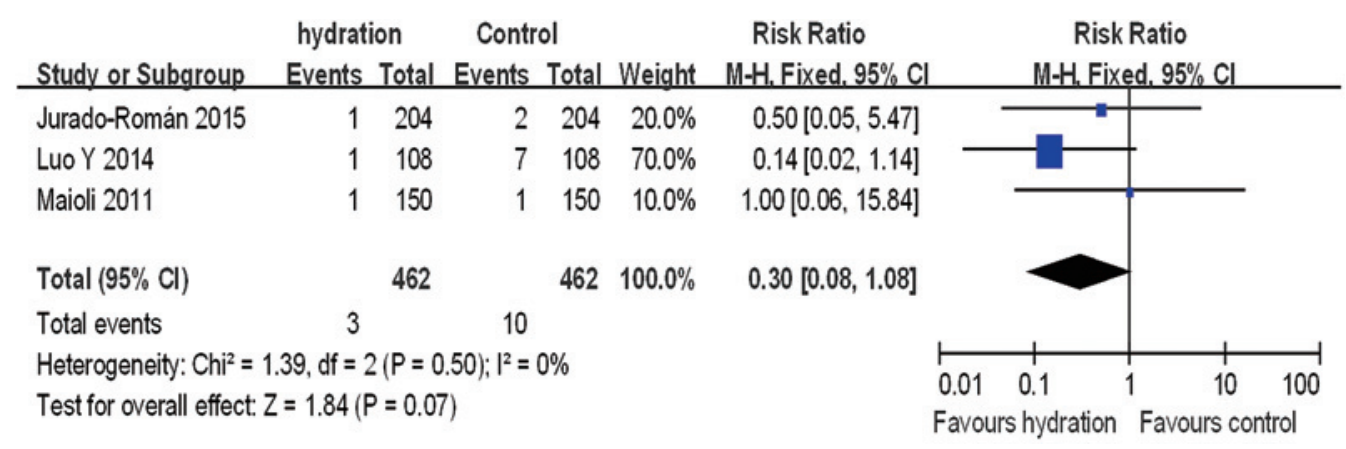

Figure 3. RR for the need for renal replacement therapy in patients who received either hydration with isotonic saline or no hydration. CI indicates confidence interval.

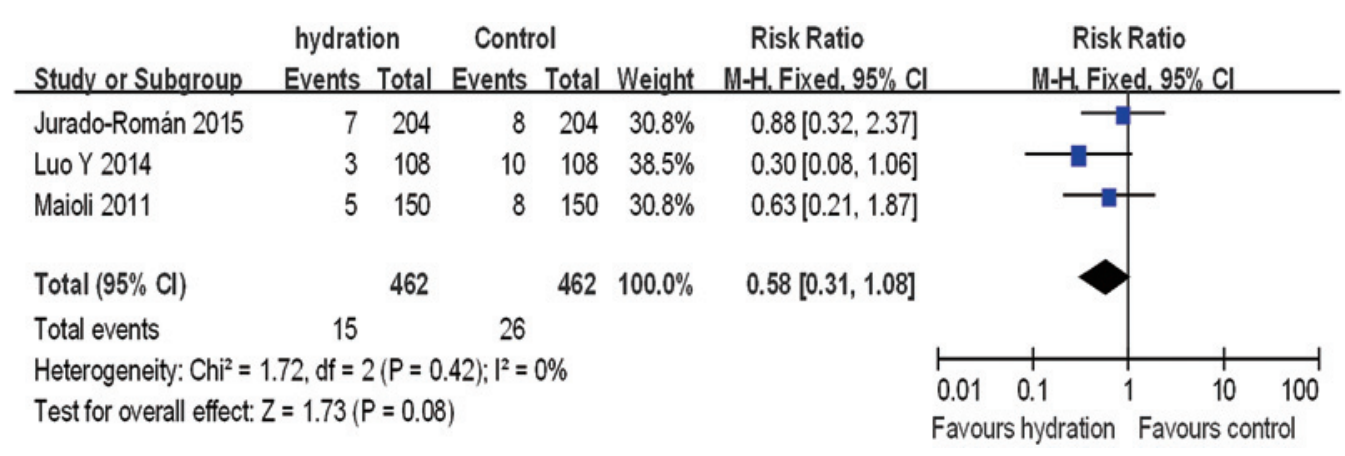

Figure 4. RR for mortality in patients who received either hydration with isotonic saline or no hydration. CI indicates confidence interval.

comes.

In conclusion, our study indicated that intravenous saline hydration during PPCI reduced the risk of CIN without significantly altering the rate of requirement for renal replacement therapy or mortality.

\section{Disclosures}

Conflicts of interest: None.

\section{References}

1. Busch SV, Jensen SE, Rosenberg J, Gögenur I. Prevention of contrast-induced nephropathy in STEMI patients undergoing primary percutaneous coronary intervention: a systematic review. J Interv Cardiol 2013; 26: 97-105.

2. Jurado-Román A, Hernández-Hernández F, García-Tejada J, et al. Role of hydration in contrast-induced nephropathy in patients who underwent primary percutaneous coronary intervention. Am J Cardiol 2015; 115: 1174-8.

3. Thayssen P, Lassen JF, Jensen SE, et al. Prevention of contrastinduced nephropathy with $\mathrm{N}$-acetylcysteine or sodium bicarbonate in patients with ST-segment-myocardial infarction: a prospective, randomized, open-labeled trial. Circ Cardiovasc Interv 
2014; 7: 216-24.

4. Liu Y, Chen JY, Huo Y, et al. RESCIND group. Aggressive hydraTion in patients with ST-Elevation Myocardial infarction undergoing Primary percutaneous coronary intervention to prevenT contrast-induced nephropathy (ATTEMPT): study design and protocol for the randomized, controlled trial, the ATTEMPT, RESCIND 1 (First study for Reduction of contraSt-induCed nephropathy followINg carDiac catheterization) trial. Am Heart J 2016; 172: 88-95.

5. Roffi M, Patrono C, Collet JP, et al. Management of acute coronary syndromes in patients presenting without persistent STsegment elevation of the European Society of Cardiology. 2015 ESC Guidelines for the management of acute coronary syndromes in patients presenting without persistent ST-segment elevation: task force for the management of acute coronary syndromes in patients presenting without persistent ST-segment elevation of the European Society of Cardiology (ESC). Eur Heart J 2016; 37: 267-315

6. Li YL, Wan Z, Lu WL, Wang JH. Comparison of sirolimus- and paclitaxel-eluting stents in patients undergoing primary percutaneous coronary intervention for ST-elevation myocardial infarction: a meta-analysis of randomized trials. Clin Cardiol 2010; 33: 583-90.

7. Navaneethan SD, Singh S, Appasamy S, Wing RE, Sehgal AR. Sodium bicarbonate therapy for prevention of contrast-induced nephropathy: a systematic review and meta-analysis. Am J Kidney Dis 2009; 53: 617-27.

8. Luo Y, Wang X, Ye Z, et al. Remedial hydration reduces the incidence of contrast-induced nephropathy and short-term adverse events in patients with ST-segment elevation myocardial infarction: a single-center, randomized trial. Intern Med 2014; 53: 2265-72.
9. Maioli M, Toso A, Leoncini M, Micheletti C, Bellandi F. Effects of hydration in contrast-induced acute kidney injury after primary angioplasty. A randomized, controlled trial. Circ Cardiovasc Interv 2011; 4: 456-62.

10. Lin KY, Wu ZY, You ZB, et al. Pre-procedural n-terminal pro-b type natriuretic peptide predicts contrast-induced acute kidney injury and long-term outcome in elderly patients after elective percutaneous coronary intervention. Int Heart J 2018; 59: 92634.

11. Brar SS, Hiremath S, Dangas G, Mehran R, Brar SK, Leon MB. Sodium bicarbonate for the prevention of contrast induced-acute kidney injury: a systematic review and meta-analysis. Clin J Am Soc Nephrol 2009; 4: 1584-92.

12. Zoungas S, Ninomiya T, Huxley R, et al. Systematic review: sodium bicarbonate treatment regimens for the prevention of contrast-induced nephropathy. Ann Intern Med 2009; 151: 6318.

13. Maioli M, Toso A, Leoncini M, et al. Sodium bicarbonate versus saline for the prevention of contrast-induced nephropathy in patients with renal dysfunction undergoing coronary angiography or intervention. J Am Coll Cardiol 2008; 52: 599-604.

14. Brar SS, Shen AY, Jorgensen MB, et al. Sodium bicarbonate vs sodium chloride for the prevention of contrast medium-induced nephropathy in patients undergoing coronary angiography: a randomized trial. JAMA 2008; 300: 1038-46.

15. Nijssen EC, Rennenberg RJ, Nelemans PJ, et al. Prophylactic hydration to protect renal function from intravascular iodinated contrast material in patients at high risk of contrast-induced nephropathy (AMACING): a prospective, randomised, phase 3, controlled, open-label, non-inferiority trial. Lancet 2017; 389: 1312-3. 